\title{
Fascitis necrosante palpebral y shock tóxico por Streptococcus pyogenes
}

\author{
Raúl Bustos B., Gonzalo Soto G., Lilian Hickmann O. y Carlos Torres B.
}

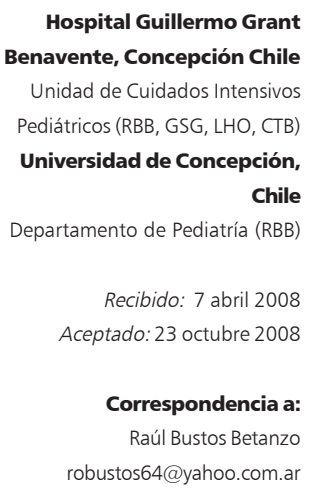

\section{Necrotizing fasciitis of the eyelids and toxic shock syndrome due to Streptococcus pyogenes}

Necrotizing fasciitis (NF) is a serious infection that compromises subcutaneous tissue, fascia, and adipose tissue, with high mortality rate and sequelae. Extremities, trunk and pelvis are the most common body sites affected. Periorbital celullitis with necrotizing fasciitis of the eyelid is rare. We report the case of a three years old child with bilateral NF of the eyelids and toxic shock syndrome secondary to Streptococcus pyogenes infection ocurring after a minor skin trauma. Early recognition leading to intensive care treatment and prompt surgical debridement were critical in the favourable outcome of the child.

Key words: Necrotizing fasciitis, pediatrics, Streptococcus pyogenes.

Palabras clave: Fascitis necrosante, pediatría, Streptocccus pyogenes.

\section{Introducción}

$\mathrm{L}$ as infecciones necrosantes de los tejidos blandos constituyen un espectro de enfermedades caracterizadas por necrosis tisular fulminante y masiva, toxicidad sistémica y alta morbi-mortalidad. Clínicamente, pueden clasificarse en superficiales y profundas. En este segundo grupo se sitúan las fascitis necrosantes (FN) y las mionecrosis que involucran compromiso de la fascia y el músculo, respectivamente. Éstas, a su vez, se dividen en tipo I o polimicrobianas -corresponden a cerca de $80 \%$ de estas infecciones- y tipo II o monomicrobianas ${ }^{1}$.

La FN usualmente se localiza en las extremidades, abdomen o perineo y excepcionalmente en la región palpebral. Describimos la presentación clínica, evolución y tratamiento de un niño antes sano que cursó con una FN palpebral bilateral asociada a shock tóxico causado por Streptococcus pyogenes, secundaria a un trauma localizado. Basados en una búsqueda realizada en la base de datos Scientific Electronic Library Online en Chile (www.SCIELO.cl), esta es la primera descripción en la literatura médica nacional de una fascitis necrosante palpebral.

Caso Clínico. Varón con 3 años de edad, sin antecedentes mórbidos de importancia, que 24 horas antes de ingresar a nuestro centro presentó una contusión accidental contra el respaldo de la cama en la zona periorbitaria derecha, por lo que acudió a consultorio, donde se le realizó una curación y prescribió tratamiento con anti-inflamatorios (diclofenaco sódico). En las horas siguientes cursó con fiebre, compromiso de conciencia y vómitos, siendo derivado a la unidad de emergencia de nuestro hospital. Al examen físico se describió un paciente de aspecto tóxico, con los siguientes signos vitales: frecuencia cardíaca: 130/min, frecuencia respiratoria: 49/min, presión arterial: 77/55 $\mathrm{mm} \mathrm{Hg}, \mathrm{T}^{\circ}$ rectal: $37,8^{\circ} \mathrm{C}$, escala de Glasgow neurológico: 14. Destacaba un gran edema periorbitario bilateral con eritema y calor en la zona palpebral y en la mejilla derecha (Figura 1). Se hospitalizó con el diagnóstico de celulitis preseptal, iniciándose tratamiento con cloxacilina endovenosa.

A las dos horas de ingresado evolucionó con hipotensión arterial refractaria al aporte enérgico de volumen y aumento del compromiso de conciencia por lo que se trasladó a la UCI pediátrica. Allí fue intubado y conectado a ventilación mecánica optimizándose la administración de volumen e iniciando soporte con fármacos inotrópicos y vasoactivos (dobutamina, noradrenalina) a través de un acceso venoso central. Entre los exámenes de laboratorio destacó: hemoglobina 7,6 $\mathrm{g} \%$, hematocrito $22,8 \%$, leucocitos $4.300 / \mathrm{mm}^{3}$, plaquetas $125.000 / \mathrm{mm}^{3}$, PCR $164 \mathrm{mg} / \mathrm{L}$, albuminemia 1,1 $\mathrm{gr} / \mathrm{dl}$, calcemia $5,6 \mathrm{meq} / \mathrm{L}$, protombinemia $21 \%, \mathrm{pH} 7,2$, EB $-12,2$ y lactacidemia $3,2 \mathrm{mmol} / \mathrm{L}$. Una TAC cerebral reveló un aumento de volumen en los tejidos blandos de la región frontal y presencia de gas en el área periobitaria derecha (Figura 2). El paciente cursó con 


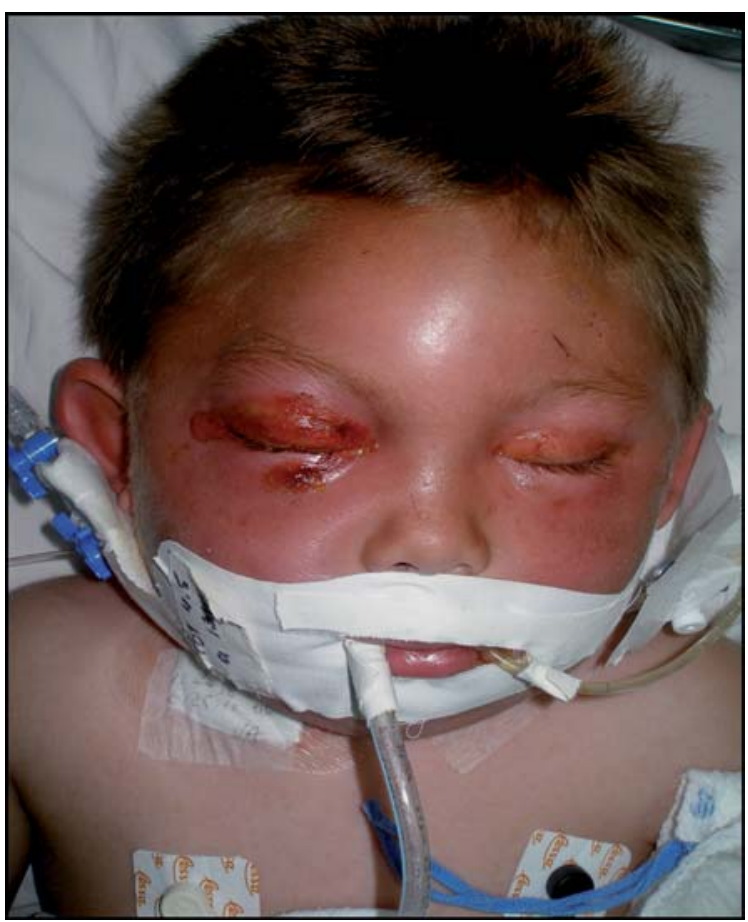

Figura 1. Aspecto del paciente al ingreso a UCl. Se destaca el aumento de volumen en la región frontal y peri orbitaria bilateral.

falla multiorgánica expresada en hipotensión arterial persistente, signos de distress respiratorio agudo y compromiso de la función hepática. Por la rapidez en la instalación del cuadro clínico, la presencia de falla multiorgánica y la aparición de un exantema maculopapular se sospechó la existencia de un shock tóxico por $S$. pyogenes ${ }^{2}$ iniciándose tratamiento sistémico con penicilina más clindamicina que se mantuvo durante 10 días. También se administró inmunoglobulina endovenosa en dosis de $2 \mathrm{gr} / \mathrm{kg}$ fraccionada en tres días. De los hemocultivos se aisló $S$. pyogenes. A las 24 horas de ingresado a la UCI aparecieron zonas de colección y necrosis de ambos lagos palpebrales, de predomino derecho, y una colección geniana lo que motivó la realización de un aseo quirúrgico y desbridamiento de ambas regiones palpebrales superiores y colocación de drenajes orales, procedimiento que fue efectuado bajo anestesia general (Figura 3). Un segundo aseo quirúrgico fue realizado a las 72 horas de ingreso (Figura 4). Los cultivos obtenidos durante la intervención y de los drenajes fueron negativos. En la TAC de órbitas de control no se observó proptosis, alteración en el trayecto del nervio óptico ni colecciones piógenas. Al quinto día de ingreso se apreciaba disminución del edema, sin secreción de pus activa, no se observaban nuevos tejidos necróticos y se mantenía con drenajes activos. El curso clínico

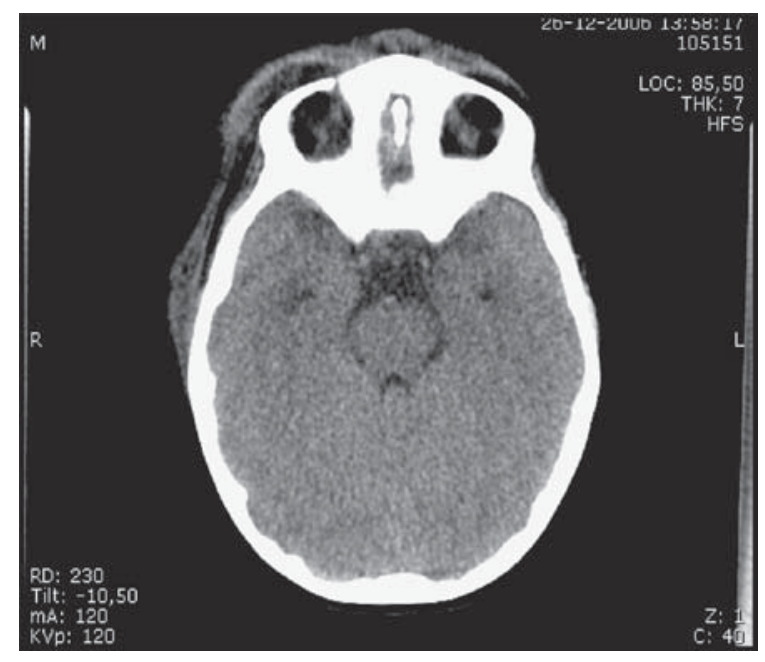

Figura 2. TAC cerebral sin medio de contraste. Se destaca el aumento de volumen de tejidos blandos. Presencia de gas en el área periorbitaria derecha.

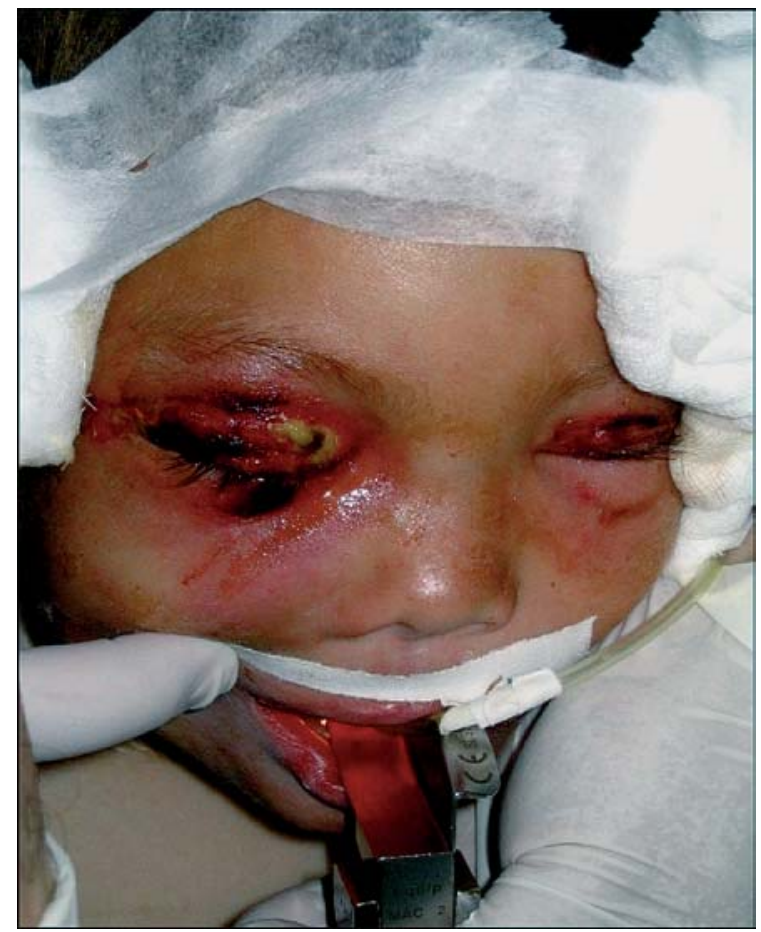

Figura 3. Necrosis palpebral bilateral con secreción de pus a derecha.

posterior fue favorable, apreciándose normalización gradual de la función cardio-respiratoria. Se mantuvo en ventilación mecánica durante cinco días y con apoyo vasoactivo por cuatro días. Egresó de la UCI a los ocho días y su alta hospitalaria ocurrió en el día 20 (Figura 5), demorada por necesidad de su atención quirúrgica y una situación social familiar precaria. En los controles posteriores realizados por equipo oftalmológico el paciente no presentó secuelas visuales. 
Figura 4. Aspecto del paciente con posterioridad a desbridamiento palpebral.

Figura 5. Aspecto del paciente previo al alta.

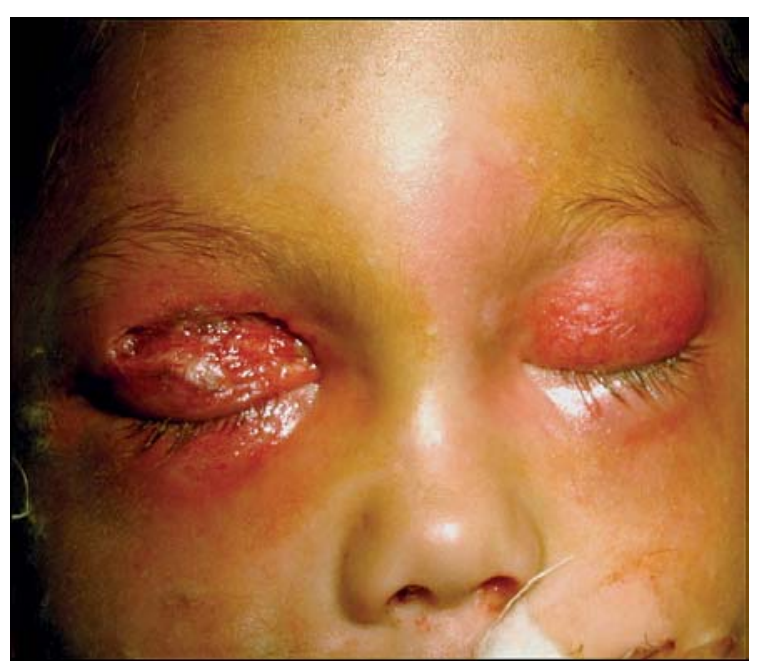

dariamente a picaduras de insecto, infecciones respiratorias altas o con posterioridad a procedimientos como dacrio-cisto-rinostomía o blefaroplastía por láser o quirúrgica ${ }^{5-8}$. Una comunicación reciente advierte sobre la aparición tardía de FN palpebral en pacientes en los que se ha realizado una rinoplastía utilizando una prótesis de silicona ${ }^{9}$.

En más de $60 \%$ de los casos, los agentes causantes de esta particular presentación de FN corresponden a S. pyogenes y Staphylococcus aureus; también se han reportado casos secundarios a infección por Haemophilus sp y Pseudomonas sp. Recientemente ha sido publicado un caso de FN palpebral causado por Moraxella catarrhalis ${ }^{10}$.

Una de las principales razones para explicar la alta mortalidad de los pacientes con FN está dada por la omisión en reconocer precozmente esta condición debido a la escasez de signos cutáneos específicos en su evolución temprana. Inicialmente, la FN palpebral es difícil de distinguir de la celulitis preseptal. La rápida progresión en 48 a 72 horas, con edema inflamatorio, a una eventual cianosis y decoloración violácea del tejido involucrado son claves para plantear el diagnóstico. La FN se presenta con la piel roja pálida y tensa. Existe dolor a medida que la infección se propaga por el tejido subcutáneo entre los planos de las fascias. Dentro de uno a dos días la piel se vuelve cianótica y gris azulada con bordes eritematosos e irregulares. La gangrena cutánea se desarrolla dentro de cuatro a cinco días, con supuración a partir del $8^{\circ}$ a $10^{\circ}$ días. En comparación a otras áreas del cuerpo, la FN de la zona palpebral tiene un curso clínico diferente; la delgada piel del párpado presenta una muy buena irrigación lo que permite que la infección de la piel sea más notoria, en tanto que su diseminación ocurre de manera profunda. El intervalo entre la aparición de los síntomas y el tratamiento ha sido reportado en alrededor de tres días (mediana: 2; rango 0-28), lo cual también fue observado en nuestro paciente ${ }^{11,12}$.

Otra coincidencia con nuestro caso es que la FN palpebral puede comprometer a ambos ojos debido a que el tejido subcutáneo situado en la zona nasal ofrece poca resistencia a la diseminación de la infección. De los casos publicados, $45 \%$ presentaban compromiso bilateral ${ }^{13}$.

En general, la FN tiene un mal pronóstico. La forma localizada en párpados tiene una mortalidad de $10 \%$. Cuando la FN compromete la porción baja de la cara y el cuello, se ha descrito una mortalidad de $32 \%$, la que es atribuida a diseminación de la infección a estructuras vitales del cuello y la cavidad torácica. Otra complicación reportada es la ceguera por oclusión de la arteria retiniana, que en nuestro paciente se descartó en los controles oftalmológicos ulteriores ${ }^{14}$. 
El tratamiento incluye el uso de antimicrobianos sistémicos y el desbridamiento quirúrgico. En las infecciones invasoras causadas por $S$. pyogenes se recomienda el uso de un antibacteriano $\beta$ lactámico asociado a clindamicina. Recomendamos que, en casos similares al presentado, se considere la cobertura antimicrobiana contra $S$. pyogenes; en el presente caso, el tratamiento empírico inicial sólo consideró cobertura anti-estafilocóccica. Por otro lado, a pesar de que en la FN palpebral algunos autores han propuesto una estrategia conservadora, aplicable a pacientes cuya enfermedad es autolimitada y sin compromiso sistémico, en la mayoría de los casos se opta por efectuar desbridamiento quirúrgico junto a la antibioterapia parenteral ${ }^{15}$. El aseo quirúrgico debe ser realizado de manera urgente a fin de limitar la diseminación de la infección y preservar la mayor cantidad de tejido normal posible para facilitar los procedimientos reconstructivos posteriores.

En resumen se presenta un caso poco habitual de
FN, con localización palpebral, causada por $S$. pyogenes, donde la sospecha precoz, el tratamiento médicoquirúrgico enérgico, junto a la reanimación en UCI, permitió una evolución favorable y sin secuelas graves.

\section{Resumen}

La fascitis necrosante (FN) es una infección grave de los tejidos subcutáneos, localizada más frecuentemente en extremidades, tronco y pelvis. El compromiso de la cara y la región palpebral es inusual. Reportamos el curso clínico de un niño de tres años, previamente sano, que presentó una FN palpebral bilateral asociado a shock tóxico por Streptococcus pyogenes, secundaria a un trauma localizado. El paciente requirió tratamiento en cuidado intensivo con resucitación enérgica, antimicrobianos, inmunoglobulina intravenosa y desbridamiento quirúrgico precoz, lo que permitió su evolución favorable.

\section{Referencias}

1.- Cainzos M, González-Rodríguez F. Necrotizing soft tissue infections Curr Opin Crit Care 2007; 13: 433-9.

2.- The Working Group on Severe Streptococcal Infections. Defining the group A streptococcal toxic shock syndrome. Rationale and consensus definition. JAMA 1993; 269: 390-1.

3.- Childers B J, Potyondy L D, Nachreiner R, Rogers F R, Childers E R, Oberg K C, et al. Necrotizing fasciitis: A fourteen-year retrospective study of 163 consecutive patients. Am Surg 2002; 68: 109-16.

4.- Elner V, Demirci H, Nerad J, Hassan A. Periocular necrotizing fasciitis with visual loss: Pathogenesis and treatment. Ophthalmology 2006; 113: 2338-45.

5.- Balaggan K, Goolamali S. Periorbital necrotizing fasciitis after minor trauma. Graefe's Arch Clin Exp Ophthalmol 2006; 244: 268-70.

6.- Costet-Fighiera C, Lagier J, Bastiani-Griffet F, Bernard E, Gastaud P. Necrotizing fasciitis of the eyelids and orbit: A life-threatening ophthalmological emergency. J Fr Ophtalmol 2002; 25: 375-8.

7.- Suñer I, Meldrum M, Johnson T, Tse D. Necrotizing fasciitis after cosmetic blepharoplasty. Am J Ophthalmol 1999; 128: 367-8.

8.- Goldberg R A, Li T G. Postoperative infection with group beta-hemolytic Streptococcus after blepharoplasty. Am J Ophthalmol 2002; 134: 908-10.

9.- Yang K C, Kuo Y S, Wu C C. Periorbital necrotizing fasciitis and orbital apex syndrome as a delayed but emergent complication of silicone nasal augmentation.
Ann Emerg Med 2007; 49: 542-3.

10.- Brittain C J, Penwarden A, Meraza A, Verita D. Moraxella as a cause of necrotizing fasciitis of the eyelid. Eye 2006; 20: 1312-4

11.- Wong C, Wang Y. The diagnosis of necrotizing fasciitis. Curr Opin Infect Dis 2005; 18: 101-6.

12.- Anaya D, Dellinger P E. Necrotizing softtissue infection: Diagnosis and management. Clin Infect Dis 2007; 44: 705-10.

13.- Bilyk J R. Periocular infection. Curr Opin Ophthalmol 2007; 18: 414-23.

14.- Kronish JW, McLeish WM. Eyelid necrosis and periorbital necrotizing fasciitis: Report of a case and review of the literature. Ophthalmology 1991; 98: 92-8.

15.- Luksich J A, Holds J B, Hartstein M E. Conservative management of necrotizing fasciitis of the eyelids. Ophthalmology 2002; 109: 2118-22. 